Arch. Tierz., Dummerstorf 49 (2006) 4, 413-414

${ }^{1}$ Research Institute for the Biology of Farm Animals (FBN), Research Unit Molecular Biology, 18196 Dummerstorf, Germany

${ }^{2}$ Research Institute for the Biology of Farm Animals (FBN), Research Group Functional Genomics, 18196 Dummerstorf, Germany

${ }^{3}$ Institute of Animal Science, Animal Breeding and Husbandry Group, University of Bonn, Endenicher Allee 15, 53115 Bonn, Germany

EDUARD MURÁNI ${ }^{1,3}$, SIRILUCK PONSUKSILI ${ }^{2,3}$, KARL SCHELLANDER ${ }^{3}$ and KLAUS WIMMERS $^{1,3}$

\title{
Identification and characterization of an AFLP marker associated with carcass composition in the pig (Brief report)
}

(Identifizierung und Charakterisierung eines AFLP-Markers für Schlachtkörpermerkmale beim Schwein)

Background: Amplified Fragment Length Polymorphism (AFLP) is a DNA fingerprinting technique that allows genotyping of DNA polymorphisms, mainly single nucleotide substitutions (SNP), across the whole genome with high multiplex ratio and without the need of their prior knowledge. We have successfully combined AFLP with selective genotyping for mapping of quantitative trait loci (QTL) in a $F_{2}$ resource population based on Duroc and Berlin Miniature pig (DUMI) (WIMMERS et al., 2002). Here we report application of this approach for the identification of loci associated with carcass composition in a herd of commercial German Landrace pigs.

\section{Procedures:}

Primer sequences:

Vec15: 5'-AGG CAC ATG ACA ACG CTG AG-3'

Aspec2: 5'-AGT TGT GGG GTT TGA CCT GTG-3'

Animals and AFLP analysis:

Samples and phenotype records $(\mathrm{n}=239)$ for AFLP and association analysis were obtained from castrates of the German Landrace (LR) kept at the performance test station Frankenforst, University of Bonn. Selection of animals for AFLP selective genotyping was based on loin muscle area (LMA). The population mean LMA was $42.5 \pm 4.5 \mathrm{~cm}^{2}$, whereas animals in the low group $(\mathrm{n}=20)$ had a mean LMA of 35.6 $\mathrm{cm}^{2}$ and animals in the high group $(\mathrm{n}=20)$ had a mean LMA of $49.7 \mathrm{~cm}^{2}$. Other traits included in the association analysis were carcass lean content estimated using "Bonner formula” (LEANP), lean content estimated using Fat-O-Meater (FOM) and loin fat depth (BFLO). The AFLP analysis was performed using TaqI- and EcoRI-adapters and primers with one and three selective nucleotides for pre- and selective amplification, respectively, essentially as described before (WIMMERS et al., 2002). Conversion of AFLP to sequence tagged site (STS) markers was done by vectorette PCR (Sigma, Taufkirchen, Germany).

Genotyping, mapping and association analysis:

For genotyping of the marker STS-Bo2 a PCR $\left(\mathrm{T}_{\mathrm{A}}=60{ }^{\circ} \mathrm{C}\right)$ was performed using the primers Vec15 and Aspec2 and restriction digestion using 6U of TthHB8I (Amersham PharmaciaBiotech, Braunschweig, Germany) at $65^{\circ} \mathrm{C}$ overnight. Physical mapping of STS-Bo2 was achieved by screening of the IMpRH panel using the same primers in a stepdown PCR $\left(\mathrm{T}_{\mathrm{A}}=72-60{ }^{\circ} \mathrm{C}\right)$ and by analysis of the results using multipoint analysis option of the IMpRH mapping tool (http://www.toulouse.inra.fr). For linkage mapping 17 informative families ( $n=287$ ) of the DUMI $F_{2}$ resource population were genotyped. Multipoint linkage map was established using the BUILD option of the CRIMAP 2.4 
package. Distribution of the AFLP markers was analyzed by Fisher exact test in a $2 \times$ 2 contingency table (Proc FREQ, SAS V6.12) and the association of STS-Bo2 with carcass traits in the whole LR herd was analyzed using a mixed linear model (Proc MIXED) including genotype as a fixed effect, sire and dam nested within sire as random effects and slaughter weight as a covariate.

Results: Using 8 AFLP primer combinations the distribution (presence/absence) of 62 AFLP markers between the high and low LMA groups was analyzed. Distribution of three markers was significantly different between the high and low group at the level $\mathrm{p}<0.05$. The most significant $(\mathrm{p}=0.000013$ ) marker $E-A G T / T-A A C 1$, enriched in the high group, was converted to a codominant marker STS-Bo2 (AccNr.DQ787147). Using vectorette PCR a C>T SNP underlying the E-AGT/T-AAC1 polymorphism was identified and on its basis a TthHB8I PCR-RFLP was designed. The wild type $\mathrm{C}$ allele, enriched in the high group (high $=0.65 \mathrm{vs}$. low $=0.20$ ), segregated with a frequency of 0.44 in the whole LR herd. On the level of the whole herd the association with LMA could not be confirmed, however a trend corresponding to the selective genotyping was observed with the CC homozygous animals having $1.3 \mathrm{~cm}^{2}$ larger LMA compared to TT homozygous animals $(\mathrm{p}=0.27)$. A similar tendency was observed for lean content (CC vs TT: LEANP $+0.78 \%$, $\mathrm{p}=0.15$; FOM $+1.6 \%$, $\mathrm{p}=0.06$ ). The positive trend in lean content associated with the $\mathrm{C}$ allele is explained by its significant negative association with BFLO (CC vs TT: $-0.17 \mathrm{~cm}, \mathrm{p}=0.04$ ). The sequence of STSBo2 shows homology (78\% over 332bp) to an intergenic sequence on human chromosome $11(\sim 34.8 \mathrm{Mb})$. Based on current human-pig comparative maps the predicted location of STS-Bo2 is on porcine chromosome 2 (SSC2), interval SW1686SW942. This is in perfect agreement with the assignment of STS-Bo2 to markerinterval SW1686-SW1564 on SSC2 using IMpRH panel (Vector: 0000000000 $\begin{array}{lllllll}0010010101 & 1000000101 & 1000000000 & 0100100000 & 0100000001 & 0101000000\end{array}$ $000000000100000000010000000101001000010001011010)$. This assignment is further supported by the linkage mapping of STS-Bo2 to the interval SW240-(23)-STSBo2-(32.1)-SW1564 (in parentheses the map distance in Kosambi cM) in the DUMI population. Intriguingly, a QTL for the diameter of fast twitch muscle fibers, which to a large extent determine LMA, was mapped to this region by WIMMERS et al. (2006). Furthermore, several QTL for carcass composition traits were mapped in divergent pig crosses in this region as well (http://www.animalgenome.org/QTLdb). Our results indicate, that the gene(s) underlying these QTL segregate in LR pigs and may be exploited in marker assisted selection.

\section{References}

WIMMERS, K.; MURANI, E.; PONSUKSILI, S.; YERLE, M.; SCHELLANDER, K.: Detection of quantitative trait loci for carcass traits in the pig by using AFLP. Mamm. Genome 13 (2002), 206-210

WIMMERS, K.; FIEDLER, I.; HARDGE, T.; MURANI, E.; SCHELLANDER, K.; PONSUKSILI, S.: QTL for microstructural and biophysical muscle properties and body composition in pigs. BMC Genetics 7 (2006), 15-28 